\title{
Diagnostic and treatment dilemmas in severe course of multicentric Castleman disease
}

\author{
Michalina Tamowicz ${ }^{\# 1}$, Agnieszka Piekarska ${ }^{\# 2}$ (D), Michał Kunc ${ }^{3}$ (D), Magdalena Dutka² (D), \\ Ewa Zarzycka $^{2}$ (D), Wojciech Biernat ${ }^{3}$ iD, Maria Bieniaszewska² (D, Jan Maciej Zaucha² iD \\ ${ }^{1}$ Medical University of Gdańsk, Gdańsk, Poland \\ ${ }^{2}$ Department of Hematology and Transplantology, Medical University of Gdańsk, Gdańsk, Poland \\ ${ }^{3}$ Department of Pathomorphology, Medical University of Gdańsk, Gdańsk, Poland
}

${ }^{\#}$ Equally contributing authors

\section{Introduction}

Castleman disease $(C D)$ is a rare benign lymphoproliferative disorder occurring in two forms: unicentric (UCD), described by Castleman in 1956, and multicentric (MCD), described in 1978 by Gaba [1, 2]. In UCD, 50\% of patients remain asymptomatic, while MCD can manifest as systemic inflammation resulting from excessive production of proinflammatory cytokines, especially interleukin-6 (IL-6) [3]. Subtypes of MCD include idiopathic MCD (iMCD) with TAFRO (thrombocytopenia, anasarca/ascites, reticulin bone marrow fibrosis, renal failure, organomegaly) and non-TAFRO clinicopathological variants. Both subtypes may have overlapping clinical features, making their distinction very difficult. We herein present such an interesting case.

\section{Case description}

In September 2020, a 50-year-old woman was admitted with a 3-week history of malaise, fever, stabbing pain in the right hypochondrium, itching of forearms, loss of appetite, and general swelling. She had had cardiac infarction at the age of 47, 15 pack-years of smoking, and a family history of systemic lupus erythematosus (SLE). Initial laboratory testing showed elevated C-reactive protein [CRP; $196.3 \mathrm{mg} / \mathrm{L}$; normal range $(\mathrm{N})<5]$, thrombocytosis $(714 \mathrm{G} / \mathrm{L})$ and leukocytosis $(20.75 \mathrm{G} / \mathrm{L})$ with normal hemoglobin $(12.9 \mathrm{~g} / \mathrm{dL})$. Computed tomography (CT) revealed pleural effusion, hepatosplenomegaly, and mediastinal lymphadenopathy up to $26 \mathrm{~mm}$ (Figure 1A, B).
Despite sequential empiric antibiotics, the patient's general condition deteriorated. She developed dyspnea, progressive anasarca, ascites, and worsening renal function (creatinine $3.15 \mathrm{mg} / \mathrm{dL}$ ), requiring continuous renal replacement therapy.

Differential diagnoses included infections, autoimmune disorders, and neoplasms. The work-up for multiple viruses [human herpesvirus-8 (HHV-8), human immunodeficiency virus (HIV), Epstein-Bárr virus (EBV), cytomegalovirus (CMV)] was negative. Serum amyloid $A$ was $1,440 \mathrm{mg} / \mathrm{L}(\mathrm{N}<6.4 \mathrm{mg} / \mathrm{L}$ ). Autoantibody profile revealed the presence of nonspecific anti-nuclear antibodies (ANA)-Hep2 (1:2,560), anti-Sjögren syndrome antibodies (SSA)-Ro52 antibodies (+++), positive lupus anticoagulant with negative anticardiolipin, and anti-beta ${ }_{2}$-glicoprotein antibodies, but she did not meet the criteria of any autoimmune disease.

A bone marrow biopsy was not diagnostic. Histological evaluation of lymph node biopsy revealed regressed germinal centers, overgrowth of the parafollicular zone with multiple vessels, and plasmacytic infiltration consistent with the hyaline-vascular type of CD (Figure 1.C-E). Additional tests revealed hypoalbuminemia $18 \mathrm{~g} / \mathrm{L}$ (N 35-50 g/L), increased alkaline phosphatase $202 \mathrm{U} / \mathrm{L}$ (N 39-100 U/L), and lactate dehydrogenase $254 \mathrm{U} / \mathrm{L}$ (N 125-220 U/L), with unaltered aminotransferases. Serum protein electrophoresis was normal. IgG remained within the normal range $(10.82 \mathrm{~g} / \mathrm{L})$. Serum IL-6 was elevated $152 \mathrm{pg} / \mathrm{mL}(\mathrm{N}<5.9 \mathrm{pg} / \mathrm{mL})$. Hemoglobin decreased to $8.9 \mathrm{~g} / \mathrm{dL}$. Given the patient's clinical presentation,

\footnotetext{
*Address for correspondence: Agnieszka Piekarska, Department of Hematology and Transplantology, Medical University of Gdansk, Smoluchowskiego 17 80-214 Gdansk, Poland, fax +48 5858443 50, e-mail: agnieszka.piekarska@gumed.edu.pl
}
PTHiT Copyright (c) 2021
The Polish Society of Haematologists and Transfusiologists, Insitute of Haematology and Transfusion Medicine. All rights reserved.




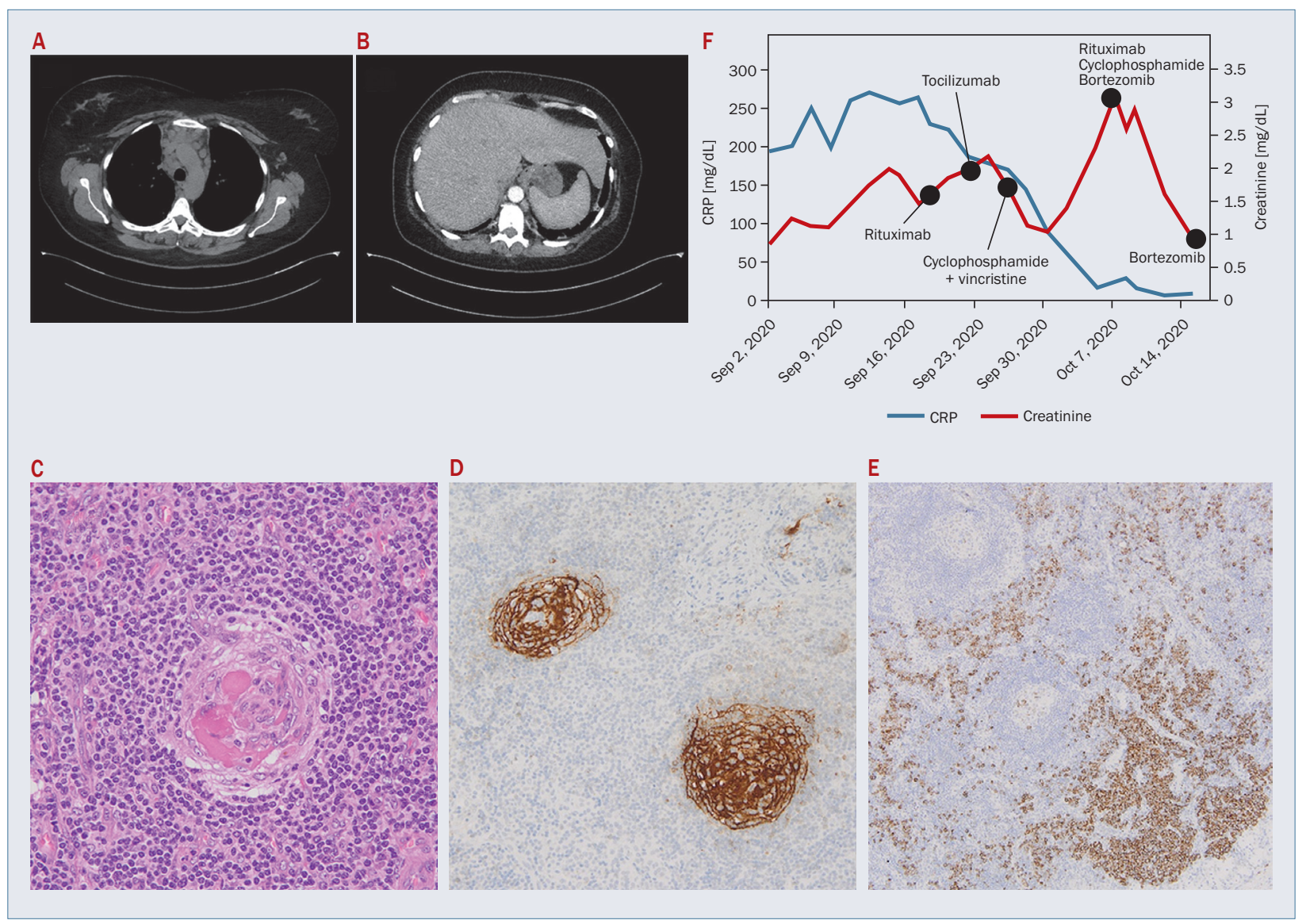

Figure 1. Chest computed tomography (CT) (A) and abdomen CT (B) performed at admission. Histological view of lymph nodes; C. Regressed germinal centers penetrated by hyalinized blood vessels surrounded by hyperplastic mantle zone. Proliferation of endothelial venules in interfollicular areas was observed; D. CD23 staining demonstrates atrophic germinal centers with prominent meshwork of follicular dendritic cells; E. Focal aggregates of CD138-positive polytypic plasma cells in interfollicular areas; F. Graph showing impact of targeted treatment administration (marked with dots) on C-reactive protein (CRP) and creatinine fluctuations during hospitalization

including non-infectious lymphadenopathy, hepatosplenomegaly, anasarca, renal failure, and histology, the findings indicated a severe course of IMCD or iMCD with TAFRO syndrome.

Treatment included initially a high dose of methylprednisolone and rituximab $\left(375 \mathrm{mg} / \mathrm{m}^{2}\right)$, followed by tocilizum$\mathrm{ab}(8 \mathrm{mg} / \mathrm{kg})$, without regression of the clinical symptoms. Therefore, cyclophosphamide and vincristine were administered, leading to a brief improvement followed by deterioration. She received a second dose of rituximab, cyclophosphamide and, to target infiltrating plasmacytes and due to immunomodulatory proprieties, we added bortezomib $\left(1.3 \mathrm{mg} / \mathrm{m}^{2}\right)$. After this combined therapy, a remarkable improvement occurred in the patient's general condition (Figure 1F). She continued the maintenance therapy with cyclophosphamide $500 \mathrm{mg}$, bortezomib $1.3 \mathrm{mg} / \mathrm{m}^{2}$ and dexamethasone $20 \mathrm{mg}$ once a week for six months at the Daily Clinic, and then treatment was discontinued. Up to now (9 months), she remains in complete remission confirmed in CT.

\section{Discussion}

The exact cause of iMCD is unknown. The overlapping clinical and pathological symptoms with autoimmune disorders such as SLE, Sjögren syndrome, and rheumatoid arthritis (RA), suggest that immune dysregulation and cytokines overproduction may contribute to iMCD [4]. The three most likely mechanisms responsible for hypercytokinemia are: 1) autoimmune driven by autoantibodies (the systemic inflammatory disease hypothesis); 2) ectopic cytokine secretion by malignant or benign cells within lymph nodes (the paraneoplastic hypothesis); and 3) viral signaling by a non-HHV-8 virus [5].

In our case, neither viral infections nor neoplasm was identified. This makes the systemic inflammatory disease hypothesis the most probable explanation of her symptoms. Specifically, the identified ANA-Hep2 and anti-SSA antibodies could induce hypercytokinemia. However, multisystemic involvement can be seen in many autoimmune disorders, and almost all lymph nodes of patients with 
RA, and $15-30 \%$ with SLE, present lesions similar to hyaline-vascular or mixed type $C D[6,7]$. In-depth diagnostics led two rheumatologists to agree on the diagnosis of $\mathrm{MMCD}$.

The diagnostic criteria for $\mathrm{M} C D$ require the fulfillment of both major criteria and at least 2/11 minor criteria [8]. Our case met both major criteria and fulfilled minor criteria, both laboratory (CRP, anemia, hypoalbuminemia, renal failure) and clinical (fever, fatigue, hepatosplenomegaly, anasarca, ascites). In TAFRO, lymphadenopathy is mild $(<1.5 \mathrm{~cm})$ with a smaller extent of plasmacytosis and myelofibrosis present in the bone marrow. Clinically, patients present with polyserositis and renal dysfunction $[9,10]$. Constitutional symptoms, hepatosplenomegaly, and renal failure are present in both TAFRO and non-TAFRO iMCD. Platelet count helps to differentiate non-TAFRO iMCD (thrombocytosis) from TAFRO (thrombocytopenia), while hypergammaglobulinemia and plasmacytic infiltration of the lymph nodes are more typical for non-TAFRO iMCD.

In our case, the presence of severe anasarca, fever, renal failure, and organomegaly suggested TAFRO-iMCD, while lymph nodes $>1.5 \mathrm{~cm}$ and thrombocytosis suggested non-TAFRO. Unfortunately, we lack data from the bone marrow. Non-TAFRO iMCD is corroborated by anemia, thrombocytosis, renal failure, and plasmacytic infiltration in lymph nodes, but not by hypergammaglobulinemia.

In summary, the whole clinical picture is fairly consistent with severe non-TAFRO iMCD. Our third line treatment combined the standard chemotherapy with bortezomib, listed as an option in the literature, which turned out to be effective. However, we cannot exclude the overlapping postponed effect of the immunotherapy targeting the IL-6 receptor.

\section{Authors' contributions}

MT - clinical analysis, writing manuscript; AP - clinical analysis, writing manuscript; $\mathrm{MK}$ - histopathological revision, microscopic images, critical revision; MD - clinical analysis, critical revision; E.Z. - clinical analysis, critical revision; WB - histopathological revision, microscopic images, critical revision; MB - clinical analysis, critical revision; JMZ - clinical analysis, writing manuscript.

\section{Conflicts of interest \\ None.}

\section{Financial support \\ None.}

\section{Ethics}

The work described in this article has been carried out in accordance with The Code of Ethics of the World Medical Association (Declaration of Helsinki) for experiments involving humans; EU Directive 2010/63/EU for animal experiments; Uniform requirements for manuscripts submitted to biomedical journals.

\section{References}

1. Castleman B, Iverson L, Menendez V. Localized mediastinal lymphnode hyperplasia resembling thymoma. Cancer. 1956; 9(4): 822-830, doi: 10.1002/1097-0142(195607/08)9:4<822::aid-cncr2820090430>3.0.co;2-4.

2. Gaba AR, Stein RS, Sweet DL, et al. Multicentric giant lymph node hyperplasia. Am J Clin Pathol. 1978; 69(1): 86-90, doi: 10.1093/ ajcp/69.1.86, indexed in Pubmed: 619617.

3. Herrada J, Cabanillas F, Rice L, et al. The clinical behavior of localized and multicentric Castleman disease. Ann Intern Med. 1998; 128(8): 657-662, doi: 10.7326/0003-4819-128-8-199804150-00010, indexed in Pubmed: 9537940.

4. Fajgenbaum DC, Shilling D. Castleman disease pathogenesis. Hematol Oncol Clin North Am. 2018; 32(1): 11-21, doi: 10.1016/j. hoc.2017.09.002.

5. Fajgenbaum DC, van Rhee F, Nabel CS. HHV-8-negative, idiopathic multicentric Castleman disease: novel insights into biology, pathogenesis, and therapy. Blood. 2014; 123(19): 2924-2933, doi: 10.1182/ blood-2013-12-545087, indexed in Pubmed: 24622327.

6. Kojima M, Motoori T, Asano S, et al. Histological diversity of reactive and atypical proliferative lymph node lesions in systemic lupus erythematosus patients. Pathol Res Pract. 2007; 203(6): 423-431, doi: 10.1016/j.prp.2007.03.002, indexed in Pubmed: 17540509.

7. Kojima M, Motoori T, Nakamura S. Benign, atypical and malignant lymphoproliferative disorders in rheumatoid arthritis patients. Biomed Pharmacother. 2006; 60(10): 663-672, doi: 10.1016/j.biopha.2006.09.004, indexed in Pubmed: 17064872.

8. Fajgenbaum DC, Uldrick T, Bagg A, et al. International, evidence-based consensus diagnostic criteria for HHV-8-negative/idiopathic multicentric Castleman disease. Blood. 2017; 129(12): 1646-1657, doi: 10.1182/blood-2016-10-746933.

9. Kawabata H, Takai K, Kojima M, et al. Castleman-Kojima disease (TAFRO syndrome): a novel systemic inflammatory disease characterized by a constellation of symptoms, namely, thrombocytopenia, ascites (anasarca), microcytic anemia, myelofibrosis, renal dysfunction, and organomegaly : a status report and summary of Fukushima (6 June, 2012) and Nagoya meetings (22 September, 2012). J Clin Exp Hematop. 2013; 53(1): 57-61, doi: 10.3960/jslrt.53.57, indexed in Pubmed: 23801135.

10. Wang HW, Pittaluga S, Jaffe ES. Multicentric Castleman disease: where are we now? Semin Diagn Pathol. 2016; 33(5): 294-306, doi: 10.1053/j.semdp.2016.05.006, indexed in Pubmed: 27296355. 